\title{
Probiotics and Diarrhea
}

\author{
Lynne V. McFarland \\ Department of Medicinal Chemistry, School of Pharmacy, University of Washington, and Department of \\ Health Services Research and Development, VA Puget Sound Health Care System, Seattle, Wash., USA
}

Probiotics have become increasing popular as a therapeutic strategy for diseases, especially those resulting from a disruption of normal microbiota [1]. The advantages of probiotics include multiple mechanisms of action, efficacy for a variety of diseases and conditions and a remarkable safety profile. Probiotics may act by producing bacteriocins against pathogens, or by competitively inhibiting the attachment of pathogens or their toxins, or by directly destroying pathogenic toxins, or by enhancing the host physiology or act as immune regulators. However, choosing the appropriate probiotic can be challenging for the physician and patient alike. The efficacy is dependent upon matching the correct probiotic strain with the appropriate indication, using sufficient doses and duration of treatment and selecting a probiotic with high quality control standards. The appropriate probiotic strain should be chosen based on evidence from welldesigned randomized controlled trials (RCT).

Of over 300 RCT published in the literature on probiotics, $19 \%$ were for the treatment or prevention of pediatric diarrhea, $16 \%$ were on the prevention of antibioticassociated diarrhea (AAD) and 6\% were for the prevention of traveler's diarrhea (TD). Pediatric diarrhea occurs in 16.5 million children under the age of 5 years per year in the USA and is a significant cause of mortality in developing countries. Although oral rehydration is the standard therapy, this does little to shorten the symptoms of diarrhea or restore the normal microbiota in the colon, which is important for the patient's recovery. Many strains of probiotics have been tested for pediatric diarrhea including Lactobacillus rhamnosus GG, Saccharomyces boulardii and mixtures of several probiotic strains. Of 54 RCT done testing 8 different single strains and different mixtures of 2-4 strains, $78 \%$ found significant effects for pediatric diarrhea. As shown in table 1 , the strength of the clinical evidence supports the use of several types of probiotic for pediatric diarrhea. For example, Pedone et al. [2] enrolled 287 children (7-32 months old) in 12 day care centers suffering from acute diarrhea and randomized them to either Actimel (L. casei DN-114 001, L. bulgaricus, Streptococcus thermophilus) or control milk formula or control yogurt for 1 month. The duration of diarrhea was significantly shortened in the probiotic group (4.3 days, $\mathrm{p}<0.05)$ compared to the control milk formula ( 8 days) and a trend was found for the control yogurt (5.3 days, $\mathrm{p}=0.06$ ).

The incidence of AAD may range from 5 to $25 \%$ depending upon the type of antibiotic and risk factors of the host. The consequences of AAD may include dehydration, longer hospitalizations, increased risk of other nosocomial infections, reduced antibiotic compliance and higher healthcare costs [3]. Of 41 RCT, 58\% showed sig-

\section{KARGER}

Fax +4161306 1234

E-Mail karger@karger.ch

www.karger.com (c) 2010 S. Karger AG, Basel

$0250-6807 / 10 / 0575-0010 \$ 26.00 / 0$

Accessible online at:

www.karger.com/anm
Lynne V. McFarland, PhD

Department of Health Services Research and Development

VA Puget Sound Health Care System

1100 Olive Way, Suite 1400, Seattle, WA 98101 (USA)

E-Maillynne.mcfarland@va.gov 
Table 1. Strength of evidence for probiotic therapy for 3 types of diarrheal diseases

\begin{tabular}{lllll}
\hline Type of disease & L. casei DN-114001 & L. rhamnosus GG & S. boulardii & L. acidophilus \\
\hline Pediatric diarrhea & +++ & ++++ & ++++ & 0 \\
AAD & + & +++ & ++++ & + \\
TD & none & + & +++ & 0 \\
\hline
\end{tabular}

Significant efficacy from: $>3$ RCT with $>100$ subjects $(++++), 2-3$ RCT with $>100$ subjects $(+++)$, $\geq 2$ RCT with $<100$ subjects $(++)$ and only 1 RCT with $>100$ subjects $(+)$; no significant efficacy found in any RCT $(0)$ or no published RCT (none).

nificant prevention of AAD by probiotics. Table 1 shows the most promising probiotic strains for the prevention of AAD. For example, Hickson et al. [4] randomized 135 inpatients given antibiotics to Actimel or placebo milkshake for the duration (plus 1 week) of the antibiotic. Significantly fewer given the probiotic mixture developed AAD (12.3\%) compared to the controls (33.9\%).

TD may also be common, especially for high-risk destinations. It can affect travelers and have a severe economic impact on tourist-related industries. Of 13 RCT for TD, 38\% showed significant prevention of TD by probiotics. For example, Kollaritsch et al. [5] randomized 3,000 Austrian tourists travelling to Turkey to either a low dose $(250 \mathrm{mg} /$ day) or a high dose (1,000 mg/day) of S. boulardii or placebo for the duration of their trip. Significantly fewer given the high probiotic dose developed TD $(29 \%$, p < $0.05)$ compared to the controls (39\%), but no effect of the low dose was found (34\%).

The use of probiotics has an excellent safety profile, as none of these RCT documented any serious adverse reactions associated with the probiotic treatment and probiotics have been used in a wide variety of patients.

Diarrhea is a global problem and many types are amenable to probiotic therapy. However, the efficacy is both strain and disease specific. Future research should confirm small studies and expand our exploration of newer probiotic strains and mixtures.

\section{Disclosure Statement}

L.V.M. is employed by the VA Puget Sound Health Care System, Seattle, Wash., USA. The views expressed in this article are those of the authors and do not necessarily reflect the position or policy of the Department of Veterans Affairs or the US government. L.V.M. has received lecture fees from Acambis, Biocodex, Danone, Massachussetts Biologic Laboratory and Osel.

References

1 Elmer GW, McFarland LV, McFarland M: The Power of Probiotics: Improving Your Health with Beneficial Microbes. London, Haworth Press Inc, 2007.

2 Pedone CA, Bernabeu AO, Postaire ER, Bouley CF, Reinert P: The effect of supplementation with milk fermented by Lactobacillus casei (strain DN-114 001) on acute diarrhoea in children attending day care centres. Int J Clin Pract 1999;53:179-184.

3 McFarland LV: Antibiotic-associated diarrhea: epidemiology, trends and treatment. Future Microbiol 2008;3:563-578.

4 Hickson M, D’Souza AL, Muthu N, Rogers TR, Want S, Rajkumar C, Bulpitt CJ: Use of probiotic Lactobacillus preparation to prevent diarrhoea associated with antibiotics: randomised double-blind placebo-controlled trial. BMJ 2007;335:80.

5 Kollaritsch H, Holst H, Grobara P, Wiedermann G: Prevention of traveler's diarrhea with Saccharomyces boulardii. Results of a placebo-controlled double-blind study. Fortschr Med 1993;111:152-156. 